\title{
Cyclophilin A Is Overexpressed in Hepatocellular Carcinoma and Is Associated with the Cell Cycle
}

\author{
ZHAOHUA GONG ${ }^{1 *}$, CHENG CHI $^{1 *}$, XIAOJUAN HUANG ${ }^{2}$, HONGJIN CHU $^{3}$, \\ JIAHUI WANG ${ }^{3}$, FENGCAI DU ${ }^{1}$, LIXIN JIANG ${ }^{4}$ and JIAN CHEN ${ }^{1,3}$ \\ ${ }^{1}$ Department of Oncology, ${ }^{3}$ The Central Laboratory, and ${ }^{4}$ Department of Gastrointestinal Surgery, \\ the Affiliated Yantai Yuhuangding Hospital of Qingdao University, Yantai, P.R. China, \\ ${ }^{2}$ First Department of Chemotherapy, Weihai Municipal Hospital, Weihai, P.R. China
}

\begin{abstract}
Aim: To investigate the expression of cyclophilin A (CypA) in human hepatocellular carcinoma (HCC) and explore the effects of CypA on the cell cycle in HCC. Materials and Methods: CypA expression was assessed by immunohistochemistry in 48 cases of HCC tissues and paired adjacent tissues. CypA plasmid was transfected into HCC cells and the cell cycle was analyzed. Results: Positivity for CypA was higher in HCC tissues than in adjacent tissues (79.1\% vs. $12.5 \%, p<0.05)$. Positivity for CypA was significantly higher in stage III and IV HCC than in stage I and II $(p<0.05)$. Elevated CypA induced an increase of the percentage of S-phase cells (from $34.79 \%$ to $42.14 \%$ ) and a decrease of $G_{0^{-}} G_{1}$ phase cells (from $58.10 \%$ to $50.64 \%$ ). Conclusion: CypA is overexpressed in HCC and is associated with TNM stage. CypA also appears to promote the transition of the cell cycle from $G_{1}$ to $S$ phase.
\end{abstract}

Hepatocellular carcinoma (HCC) is the fifth most common cancer worldwide (1). HCC is a primary cancer of the liver and generally arises from a precursor condition such as chronic hepatitis or liver cirrhosis. The high mortality rate leads HCC to be the third leading cause of cancer-related death, with over 750,000 deaths annually (2). The incidence of $\mathrm{HCC}$ is highest in the Asia-Pacific region and is increasing in Western countries $(3,4)$. Currently, surgical resection is the main radical treatment for $\mathrm{HCC}$, however, fewer than $20 \%$ of patients with HCC are candidates for surgery due to the anatomic location, size or number of

\footnotetext{
*These Authors contributed equally to this study.

Correspondence to: Dr Jian Chen, Department of Oncology, The Affiliated Yantai Yuhuangding Hospital of Qingdao University, 20 Yuhuangding East Road, Yantai, Shandong Provence, 264000, P.R. China. Tel: +86 18660086778, e-mail: chenjianyt@163.com
}

Key Words: CypA, hepatocellular carcinoma, cell cycle. tumors, or impairment of the hepatic reserve (5). Therefore, it is of great significance to explore the underlying molecular mechanisms of HCC, as well as to identify novel therapeutic targets for HCC.

Cyclophilin A (CypA), an 18-kDa protein, is a member of the cyclophilin family. It was purified from bovine thymocytes and was originally identified as a cytosolic receptor of the immunosuppressant cyclosporin $\mathrm{A}$ (CsA) (6). CypA has been found to be overexpressed in various cancer types, including early gastric cancer (7), esophageal squamous cell carcinoma (8) and breast cancer (9). CypA enhances cell proliferation, tumor growth and metastasis (10, 11). CypA overexpression induces resistance to hypoxia and chemotherapeutic agents in $\mathrm{HCC}$ and hence inhibition of CypA can promote the apoptosis of HCC cells when combined with cisplatin (12). A recent study found that CypA expression promoted HCC cell adhesion and metastasis through up-regulating matrix metalloproteinase 3 (MMP3) and MMP9 (13). However, whether CypA can regulate the cell cycle is rarely reported.

In this study, we investigated the expression level of CypA in HCC as well as analyzing whether the expression level of CypA is correlated with the clinical pathological features of HCC. We also identified a role for CypA in the cell cycle.

\section{Materials and Methods}

Tissue samples and cell culture. Samples of HCC tissue and paired normal adjacent liver tissue were collected from 48 patients who underwent surgical resection for HCC at Yantai Yuhuangding Hospital from 2015 to 2016. The pathological status of all samples was confirmed. None of these 48 patients received radiotherapy, chemotherapy or interventional therapy before operation. The differentiation grade, TNM stage and lymph node metastasis status were determined according to the TNM classification (seventh edition) (20). The clinical pathological features of these patients are summarized in Table I. This study was approved by the Ethics Committee of Yantai Yuhuangding Hospital. Informed consent was 
obtained from all patients. SK-Hep1 cells (ATCC, Manassas, VA USA) were cultured at $37^{\circ} \mathrm{C}$ with $5 \% \mathrm{CO}_{2}$ in RPMI-1640 Medium (Life Technologies Corporation, USA) supplemented with $10 \%$ fetal calf serum (FCS; PAA Laboratories, Somerset, UK).

Immunohistochemistry. Paraffin-embedded tissue sections were sectioned, deparaffinized and rehydrated in xylene and graded alcohols. Antigen retrieval was carried out in $10 \mathrm{mM}$ citrate buffer. After endogenous peroxidase activity was blocked in $3 \% \mathrm{H}_{2} \mathrm{O}_{2}$ solution, tissue sections were incubated overnight at $4{ }^{\circ} \mathrm{C}$ in the dark with rabbit antibody to CypA (1:100 dilution; Abcam, Cambridge, $\mathrm{UK})$. The sections were then washed with phosphate-buffered saline (PBS) three times and incubated for $30 \mathrm{~min}$ at $37^{\circ} \mathrm{C}$ with biotinylated secondary antibody (Invitrogen, Paisley, UK). Finally, after treatment with $0.05 \% 3,3$ '-diaminobenzidine tetrahydrochloride (DAB), the sections were counterstained with hematoxylin. HCC cells with cytoplasmic immunoreactivity for CypA were considered positive cells. Samples incubated with PBS instead of primary antibodies were used as negative controls.

Five random fields were examined under an optical microscope for each section. The results were evaluated based on the proportion of positively stained cells. Tissues with an average percentage of $\geq 50 \%$ positive cells were classified as being positively stained (+).

Cell proliferation assay and cell-cycle analysis. The CypA plasmid and empty vector pCMV-HA (OriGene Technoloqies, Rockville, USA) were transfected into SK-Hep1 cells using Lipofectamine 2000 reagent (Invitrogen) and the expressed CypA was detected using western blot. Cells were harvested and fixed in $70 \%$ ethanol at $-20^{\circ} \mathrm{C}$ for 24 hours before being resuspended with $10 \mu \mathrm{l}$ propidium iodide (Life Technologies Inc, New York, USA.) followed by incubation at room temperature for 20 minutes. The MTT assay was used to detect cell proliferation activity. Cells were plated into 96well plates at a density of $2 \times 10^{3}$ cells per well. MTT assays were performed every day over the subsequent 6 days according to the manufacturer's instructions (Sigma, Darmstadt, Germany) and growth curves were generated. Cell-cycle distribution was analyzed on a FACSVantage SE instrument (BD Biosciences Pharmingen, San Diego, CA, USA). The experiments were repeated three times.

Statistical analysis. Experimental data was analyzed using SPSS 22.0 (SPSS Inc., Chicago, IL, USA). The correlation of CypA expression with various clinical pathological features of HCC was determined by chi-square test. The cell proliferation and cell-cycle distribution was analyzed using Student's $t$-test. All statistical tests were two-sided and a value of $p<0.05$ was considered statistically significant.

\section{Results}

CypA is overexpressed in HCC and is associated with TNM stage. The expression CypA in HCC and adjacent normal tissues was evaluated by immunohistochemical analysis. Figure 1 shows that both cancer tissue and normal tissue demonstrated some positive staining. However, the positivity rate for CypA in carcinoma tissues was $79.1 \%$ (38 out of 48), while in normal adjacent tissues it was $12.5 \%$ (six of 48) (Table II). The positivity rate for CypA in the HCC tissue was significantly higher than that in the normal adjacent tissue $(p<0.05)$.
Table I. Association between cyclophilin A (CypA) expression and clinical pathological features of hepatocellular carcinoma.

\begin{tabular}{|c|c|c|c|c|}
\hline Characteristic & $\begin{array}{l}\text { Number } \\
\text { of cases }\end{array}$ & $\begin{array}{l}\text { CypA-positive } \\
\text { rate, } \mathrm{n}(\%)\end{array}$ & Chi-square & $p$-Value \\
\hline Age & & & 0.001 & 0.975 \\
\hline$<60$ Years & 31 & $24(77.4 \%)$ & & \\
\hline$\geq 60$ Years & 17 & $14(82.4 \%)$ & & \\
\hline Gender & & & 0.117 & 0.733 \\
\hline Male & 39 & $30(76.9 \%)$ & & \\
\hline Female & 9 & $8(88.9 \%)$ & & \\
\hline Tumor size $(\mathrm{cm})$ & & & 0.043 & 0.836 \\
\hline$<5$ & 25 & $19(76.0 \%)$ & & \\
\hline$\geq 5$ & 23 & $19(82.6 \%)$ & & \\
\hline TNM stage & & & 4.243 & 0.039 \\
\hline I, II & 27 & $18(66.7 \%)$ & & \\
\hline III, IV & 21 & $20(95.2 \%)$ & & \\
\hline Edmondson grade & & & 2.318 & 0.128 \\
\hline I, II & 27 & $24(88.9 \%)$ & & \\
\hline III, IV & 21 & $14(66.7 \%)$ & & \\
\hline Tumor differentiation & & & 3.245 & 0.197 \\
\hline high & 21 & $19(90.5 \%)$ & & \\
\hline middle & 15 & $11(73.3 \%)$ & & \\
\hline low & 12 & $8(66.7 \%)$ & & \\
\hline AFP level ( $\mu \mathrm{g} / \mathrm{l})$ & & & 0.000 & 1.000 \\
\hline$<400$ & 31 & $25(80.6 \%)$ & & \\
\hline$\geq 400$ & 17 & $13(76.5 \%)$ & & \\
\hline HBSAg & & & 0.284 & 0.594 \\
\hline Positive & 43 & $35(81.4 \%)$ & & \\
\hline Negative & 5 & $3(60.0 \%)$ & & \\
\hline Liver capsule invasion & & & 0.361 & 0.548 \\
\hline Yes & 20 & $15(75.0 \%)$ & & \\
\hline No & 28 & $23(82.1 \%)$ & & \\
\hline
\end{tabular}

AFP: $\alpha$-Fetoprotein; HBSAg: hepatitis B surface antigen.

Table II. Expression of cyclophilin A (CypA) in hepatocellular carcinoma and adjacent normal tissue.

\begin{tabular}{lcccc}
\hline Tissue & $\begin{array}{r}\text { Number } \\
\text { of cases }\end{array}$ & $\begin{array}{r}\text { CypA-positive } \\
\text { rate, n (\%) }\end{array}$ & Chi-square & p-Value \\
\hline Cancer & 48 & $38(79.1 \%)$ & 42.96 & $<0.005$ \\
Adjacent normal & 48 & $6(12.5 \%)$ & & \\
\hline
\end{tabular}

Correlation analysis of clinical pathological features revealed significant differences in the rates of CypA positivity among different TNM stages. Positivity for CypA expression in stage III and IV was $95.2 \%$ (20 out of 21) which was significantly higher than that in stages I and II (66.7\% 18 of 27) $(p<0.05)$ (Table I). However, CypA expression was not significantly correlated with the patient's age, gender, tumor size, tumor differentiation degree, tumor grade, $\alpha$-fetoprotein (AFP) or hepatitis B surface antigen (HBSAg) levels (Table I). 

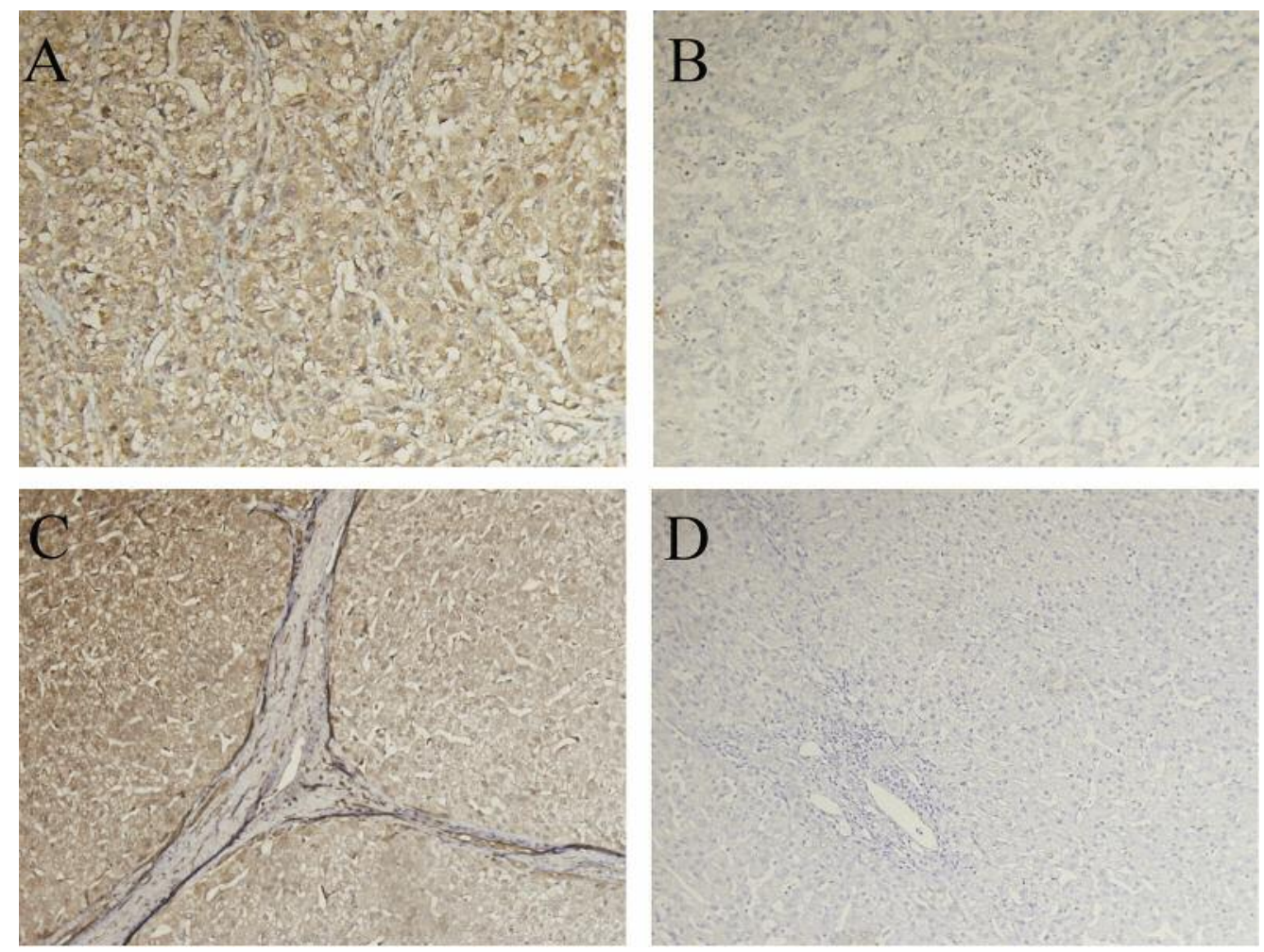

Figure 1. Immunohistochemical staining of hepatocellular carcinoma (HCC) and paired adjacent samples for cyclophilin A (CypA). A: CypA-positive HCC tissue. B: CypA-negative HCC tissue. C: CypA-positive normal adjacent tissue. D: CypA-negative normal adjacent tissue. Magnification of x100.

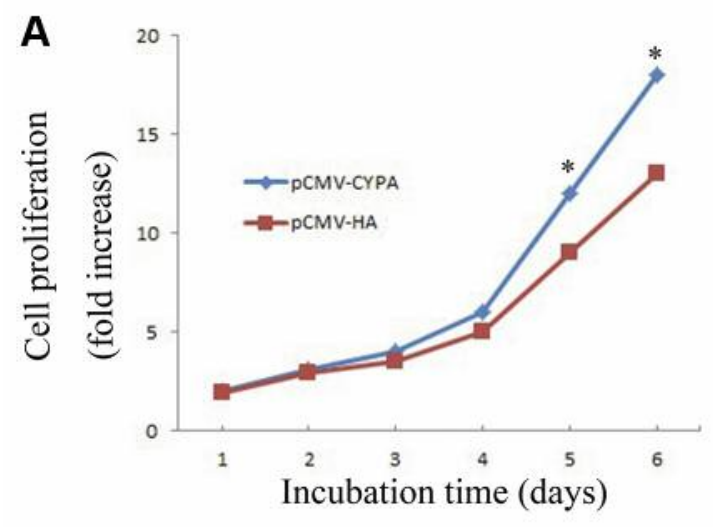

B
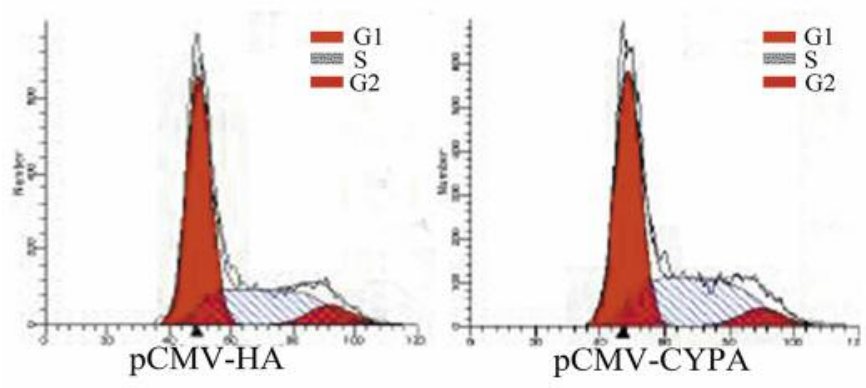

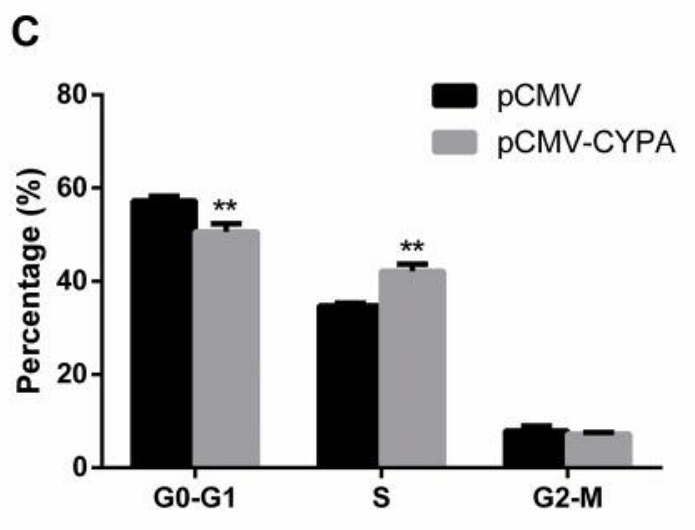

Figure 2. Regulation by cyclophilin A (CypA) on cell proliferation and cell-cycle distribution. A: Induced expression of CypA by transfection of cells with $p C M V-C Y P A$ promoted cell proliferation. By day 5 and 6, proliferation of the $p C M V$-CYPA transfected group was significantly increased compared with the control group (day 5: *p=0.015; day $6 * p=0.035$ ). B,C: The cell cycle distribution was changed when CypA was overexpressed. Compared with control cells, elevated expression of CypA induced an increase of $S$ phase cells $\left({ }^{* *} p=0.002\right)$ and a decrease of $G_{0}-G_{1}$ phase cells $(* * p=0.004)$. 
Elevated expression of CypA promotes cell proliferation through regulation of the cell cycle. To explore the role of CypA in the regulation of the cell cycle, the liver cancer cell line SK-Hep1 was transfected with CypA expression plasmid pCMV-CYPA or empty vector pCMV. As shown in Figure 2A, overexpression of CypA promoted cell proliferation. Following 5- and 6-day incubation, proliferation of the pCMV-CYPA group was significantly increased compared to the control group $(p<0.05)$.

We then compared the cell distribution using flow cytometry. As Figure 2B and C shows, the proportion of $\mathrm{S}$ phase cells in CypA-overexpressing cells was higher than in control cells $(42.14 \%$ vs. $34.79 \%, p<0.05, \quad \mathrm{~N}=3)$. Correspondingly, overexpressing CypA cells induced a decrease in cell population in $\mathrm{G}_{0}-\mathrm{G}_{1}$ phase compared with control cells ( $50.64 \%$ to $58.10 \%, p<0.05, \mathrm{~N}=3$ ). However, the percentage of cells in $\mathrm{G}_{2}-\mathrm{M}$ phase for both groups did not differ statistically.

\section{Discussion}

CypA, an important member of the cyclophilin family, is primarily located in the cytoplasm and is expressed in various tissues and organs. CypA was originally identified as a cytosolic receptor of the immunosuppressant CsA. These two proteins interact to form CypA-CsA, which can inhibit immunological rejection after organ transplantation (6). CypA, which possesses peptidyl-prolyl cis/trans isomerase and chaperone activities, not only helps maintain the correct conformation of newly synthesized proteins but can also repair misfolded proteins. CypA also has a protective effect against environmental insults (14).

During the past decade, CypA has been shown to be overexpressed in many different cancer types. CypA was highly expressed in esophageal squamous cell carcinoma by immunohistochemical analysis, and was significantly associated with tumor differentiation and shorter progression free survival times (15). Similar results were found in nonsmall cell lung cancer (NSCLC). Using quantitative reverse transcriptase polymerase chain reaction, researchers found that a NSCLC cell line expressed significantly higher levels of CypA than normal lung cell lines. Moreover, CypA stimulated cell proliferation and promoted metastasis by upregulating the activity of MMP9 in NSCLC. When the expression of CypA was down-regulated by siRNA, cell proliferation and colony formation were suppressed (10). In another study on drug resistance, CypA expression was upregulated in paclitaxel-resistant endometrial cancer cells. With RNA interference, CypA-knockdown reversed paclitaxel resistance, including inhibition of cell proliferation, induction of apoptosis and suppression of invasive capacity. This anticancer function was through suppression of the mitogen-activated protein kinase pathway
(16). In pancreatic ductal adenocarcinoma (PDAC), researchers showed that CypA is a downstream gene of hypoxia-inducible factor (HIF)- $1 \alpha$ and the high expression of both genes was significantly associated with lymph node metastasis and higher tumor stage. HIF- $1 \alpha$ directly binds to the CypA promoter regions and regulates CypA expression.

Overexpression of CypA and HIF-1 $\alpha$ promoted PDAC cell proliferation and invasion, and suppressed apoptosis (17). Overexpression of CypA was first demonstrated in HCC in 1998 (18). Subsequently, the proteomic analysis of HCC indicated that CypA may be a diagnostic marker and therapeutic target for HCC (19). Additionally, CypA expression was found to promote cancer cell adhesion and metastasis through up-regulation of MMP3 and MMP9 (13). This research provided a theoretical basis for our study.

In the present study, we detected a dramatic elevation of CypA protein level in HCC tissue specimens, which agrees well with previous studies, implying that CypA might play a crucial role in HCC malignant transformation. Furthermore, the analysis of CypA with clinical pathological features showed that the expression of CypA is significantly correlated with TNM stages, rather than age, gender, tumor size, tumor differentiation, tumor grade, AFP level and HBSAg. These results suggest that CypA may be involved in the development and progression of HCC. Subsequently, cell-cycle analysis showed that the percentage of $\mathrm{S}$ phase cells was increased while the proportion of $\mathrm{G}_{0}-\mathrm{G}_{1}$ phase cells was reduced following overexpression of CypA, indicating that CypA promotes the transition of cells in the cell cycle from $G_{1}$ to $S$ phase. This may explain why CypA can promote the growth of tumor cells.

In conclusion, CypA may become a potential factor predicting the malignancy of $\mathrm{HCC}$, as well as an attractive prognostic indicator and therapeutic target for HCC. However, the mechanism of CypA actor in HCC needs to be explored further.

\section{Acknowledgements}

This work was supported by National Natural Science Foundation of China (81071758); Shandong Science and Technology Development Project (2015GSF118142); Shandong Medicine and Health Science Technology Program (2016WS0706); China International Medical Foundation (Z20140615325); Natural Science Foundation of Shandong Province Joint Programme (ZR2016HL26, ZR2015HL069); and YantaiYuhuangding Hospital Initiative Foundation for Young Scientist (201526, 201402); Yantai Science and Technology Program (2016WS013, 2015WS018, 1605350099).

\section{References}

1 Fei Q, Shang K, Zhang J, Chuai S, Kong D, Zhou T, Fu S, Liang Y, Li C, Chen Z, Zhao Y, Yu Z, Huang Z, Hu M, Ying H, Chen Z, Zhang Y, Xing F, Zhu J, Xu H, Zhao K, Lu C, Atadja P, Xiao 
ZX, Li E and Shou J: Histone methyltransferase SETDB1 regulates liver cancer cell growth through methylation of p53. Nat Commun 6: 8651, 2015.

2 Han ZG: Functional genomic studies: insights into the pathogenesis of liver cancer. Annu Rev Genomics Hum Genet 13: 171-205, 2012.

3 Hsieh MJ, Chen MK, Chen CJ, Hsieh MC, Lo YS, Chuang YC, Chiou HL and Yang SF: Glabridin induces apoptosis and autophagy through JNK1/2 pathway in human hepatoma cells. Phytomedicine 23: 359-366, 2016.

4 Hong SW, Hur W, Choi JE, Kim JH, Hwang D and Yoon SK: Role of ADAM17 in invasion and migration of CD133expressing liver cancer stem cells after irradiation. Oncotarget 7: 23482-23497, 2016.

5 Himoto T, Kurokohchi K, Watanabe S and Masaki T: Recent advances in radiofrequency ablation for the management of hepatocellular carcinoma. Hepat Mon 12: e5945, 2012.

6 Handschumacher RE, Harding MW, Rice J, Drugge RJ and Speicher DW: Cyclophilin: a specific cytosolic binding protein for cyclosporin A. Science 226: 544-547, 1984.

7 Feng W, Xin Y, Xiao Y, Li W and Sun D: Cyclophilin A enhances cell proliferation and xenografted tumor growth of early gastric cancer. Dig Dis Sci 60: 2700-2711, 2015.

8 Feng MX, Li JP, Wang H, Shen YX, Tan LJ, Wang Q and Wang YQ: Clinical significance of cyclophilin a expression in esophageal squamous cell carcinoma. Genet Test Mol Biomarkers 19: 182-186, 2015.

9 Chevalier F, Depagne J, Hem S, Chevillard S, Bensimon J, Bertrand $\mathrm{P}$ and Lebeau $\mathrm{J}$ : Accumulation of cyclophilin A isoforms in conditioned medium of irradiated breast cancer cells. Proteomics 12: 1756-1766, 2012.

10 Qian Z, Zhao X, Jiang M, Jia W, Zhang C, Wang Y, Li B and Yue W: Down-regulation of cyclophilin A by siRNA diminishes non-small cell lung cancer cell growth and metastasis via the regulation of matrix metallopeptidase 9. BMC cancer 12: 442, 2012.

11 Obchoei S, Weakley SM, Wongkham S, Wongkham C, Sawanyawisuth K, Yao Q and Chen C: Cyclophilin A enhances cell proliferation and tumor growth of liver fluke-associated cholangiocarcinoma. Mol Cancer 10: 102, 2011.

12 Lee J: Novel combinational treatment of cisplatin with cyclophilin A inhibitors in human heptocellular carcinomas. Arch Pharm Res 33: 1401-1409, 2010.
13 Zhang M, Dai C, Zhu H, Chen S, Wu Y, Li Q, Zeng X, Wang W, Zuo J, Zhou M, Xia Z, Ji G, Saiyin H, Qin L and Yu L: Cyclophilin A promotes human hepatocellular carcinoma cell metastasis via regulation of MMP3 and MMP9. Mol Cell Biochem 357: 387-395, 2011.

14 Lee J: Role of cyclophilin a during oncogenesis. Arch Pharm Res 33: 181-187, 2010.

$15 \mathrm{Li} \mathrm{Y}$, Guo H, Dong $\mathrm{D}, \mathrm{Wu} \mathrm{H}$ and $\mathrm{Li} \mathrm{E}$ : Expression and prognostic relevance of cyclophilin $\mathrm{A}$ and matrix metalloproteinase 9 in esophageal squamous cell carcinoma. Diagnostic pathology 8: 207, 2013.

$16 \mathrm{Li} \mathrm{Z}$, Min W and Gou J: Knockdown of cyclophilin A reverses paclitaxel resistance in human endometrial cancer cells via suppression of MAPK kinase pathways. Cancer Chemother Pharmacol 72: 1001-1011, 2013.

17 Zhang H, Chen J, Liu F, Gao C, Wang X, Zhao T, Liu J, Gao S, Zhao X, Ren H and Hao J: CypA, a gene downstream of HIF1alpha, promotes the development of PDAC. PloS One 9: e92824, 2014.

18 Corton JC, Moreno ES, Merritt A, Bocos C and Cattley RC: Cloning genes responsive to a hepatocarcinogenic peroxisome proliferator chemical reveals novel targets of regulation. Cancer Lett 134: 61-71, 1998.

19 Lim SO, Park SJ, Kim W, Park SG, Kim HJ, Kim YI, Sohn TS, Noh JH and Jung G: Proteome analysis of hepatocellular carcinoma. Biochem Biophys Res Commun 291: 1031-1037, 2002.

20 Chan AC, Fan ST, Poon RT, Cheung TT, Chok KS, Chan SC, and Lo CM: Evaluation of the seventh edition of the American Joint Committee on Cancer tumour-node-metastasis (TNM) staging system for patients undergoing curative resection of hepatocellular carcinoma: implications for the development of a refined staging system. HPB 15: 439-448, 2013.
Received April 6, 2017

Revised April 20, 2017

Accepted April 24, 2017 